\title{
The effectiveness of new model of motorcycle seat with built-in lumbar support
}

\begin{abstract}
Motorcycle is getting significant attention as it is already and will be an important mode of transport in the future. However, motorcycle is a hazardous type of vehicle mode and therefore requires more efforts to enhance their safety and comfort level. As compared to car drivers, motorcyclists are more exposed to sitting hazards during the riding process. Due to increased exposures to seated postures, sitting comfort has become an important issue that demands adequate ergonomic interventions. The purpose of this study was to evaluate the effectiveness of new model of motorcycle seat with lumbar support aimed at reducing muscle discomfort among male motorcyclists. This study was conducted among male motorcyclists at University Putra Malaysia (UPM). 94 subjects were participated, with 47 subjects for each control and experimental group. Each subject was asked to sit for 2 hours on motorcycle in two different sessions. At every 15 minutes interval, subjects were required to evaluate their discomfort level on Borg's scale CR-10 questionnaire. Results in this study found that lumbar support (prototype) shows a significant effect towards certain body parts particularly on the neck, shoulder, upper back, arms and also lower back. However, there were no significant effect were found on the buttock, thigh, knee, calf and ankles. In conclusion, this new model of motorcycle seat with lumbar support can help to reduce discomfort level towards motorcyclists especially during prolonged riding process.
\end{abstract}

Keyword: Motorcycle; Prolonged riding; Muscle discomfort; Lumbar support 